\title{
FEATURES OF THE ULTRASTRUCTURE OF THE SKIN OF WHITE RATS 60 AND 90 DAYS AFTER MODELING OF PORTAL HYPERTENSION
}

DOI: 10.36740/WLek202109130

\author{
Nazar R. Hrytsevych ${ }^{1}$, Volodymyr V. Vereschaka ${ }^{2}$ \\ 'DEPARTMENT OF SURGICAL DISCIPLINES AND EMERGENCIES, FACULTY № 2, HIGHER EDUCATIONAL COMMUNAL INSTITUTION OF THE LVIV REGIONAL \\ COUNCIL "ANDREI KRUPINSKY LVIV MEDICAL ACADEMY", LVIV, UKRAINE \\ 2DEPARTMENT OF FUNDAMENTAL MEDICINE, EDUCATIONAL AND SCIENTIFIC CENTER "INSTITUTE OF BIOLOGY AND MEDICINE”, TARAS SHEVCHENKO \\ NATIONAL UNIVERSITY OF KYIV, KYIV, UKRAINE
}

\begin{abstract}
The aim: The aim of the research was to study the features of the ultrastructure of the skin of white rats 60 and 90 days after modeling of portal hypertension. Materials and methods: The investigations were performed on male white nonlinear rats. The animals of the first (control) group underwent a sham operation. In rats of the second group, portal hypertension was simulated by applying a ligature to the portal vein. 60 and 90 days after the start of the experiment, a biopsy was taken from the anterior abdominal wall for electron microscopy research.

Results: It was shown that skin in intact animals (control group) was of typical structure before beginning of experiment and 60 and 90 days after a sham operation. 60 days after the start of the experiment, we found severe edema of the basal and spinous layer of the epidermis, breakdown of intercellular contacts, edema of endotheliocytes, their perivascular edema, leukocytes infiltration, edema of mitochondria and lysosomes. 90 days after the start of the experiment, ultrastructural changes in the skin were more pronounced. Ultrastructural changes of microvessels were characterized by narrowed lumen. Endothelial cells had significant edema.

Conclusions: The data obtained should be taken into account when performing surgical interventions to prevent complications in the formation of the scar.
\end{abstract}

KEY WORDS: portal hypertension, skin, ultrastructure

Wiad Lek. 2021;74(9 p.l):2197-2201

\section{INTRODUCTION}

Regenerative processes in the skin - one of the current problems of modern medicine, as noted in a number of articles [1]. Violation of regenerative processes leads to the formation of keloids and hypertrophic scars [2]. Such scars are characterized by uncontrolled growth and excessive collagen formation [3].

Another important area in the study of regenerative processes in the skin after surgery, especially after plastic surgery, is the formation of hematomas [4-6]. What do keloids, hypertrophied scars and hematomas have in common after surgery? We believe that this is increased pressure in the vessels of the skin. Really, the most common cause of hematoma is hypertension [7]. On the other hand, when we talk about fibrotic diseases, we must provide data on the role of the local renin-angiotensin system, which modulates collagen production and wound healing $[8,9]$. Captopril, enalapril, ramipril, lisinopril, fosinopril belong to angiotensin-converting enzyme inhibitors which are widely used in the treatment of hypertension [10]. They inhibit the formation of angiotensin II and its action in tissues [11]. Niazi et al. established that AT1 receptor concentrations in patients with keloids were increased in comparison with normal group and group of patients with hypertrophic scar [9]. They also showed that concentration of angiotensin II was higher in the group of patients with hypertrophic scar in comparison with normal group and group of patients with keloids. The authors concluded that concentration of angiotensin II and AT1 receptors stimulate the formation of keloid and hypertrophic scar and angiotensin-converting enzyme inhibitors can be used for prevention and treatment of keloids and hypertrophic scars [9].

So, for checking such hypothesis and investigation of mechanism of keloid and hypertrophic scar we need to have the good model of high pressure in blood vessels of the skin on animals. Formerly we found the changes in the microcirculation and ultrastructure of the skin of the rats 30 days after portal hypertension [Article in press]. It is important to investigate these changes in dynamic and choose the time for further study of wound healing.

\section{THE AIM}

The aim of the research was to study the features of the ultrastructure of the skin of white rats 60 and 90 days after modeling of portal hypertension. 


\section{MATERIALS AND METHODS}

All experiments were carried out according to the European Council Directive on 24 November 1986 for Care and Use of Laboratory Animals (86/609/EEC), approved by First National Congress for Bioethics (September 2001), and approved by the the Ethical Committee of Educational and Scientific Center "Institute of Biology and Medicine", Taras Shevchenko National University of Kyiv, Kyiv, Ukraine.

The investigations were performed on 20 male white nonlinear rats aged 5 months which were randomly divided into 2 groups of 10 animals in each. In rats of the second group, portal hypertension was simulated by applying a ligature to the portal vein according to the conventional method [12, 13]. After 30, 60 and 90 days, under local anesthesia anterior abdominal wall biopsies were taken. Material for electron microscopy research (20 skin bioptates, 10 in each group) were chopped into pieces up to 1 $\mathrm{mm}$ thick and fixed during $1.5-2$ hours at $30^{\circ} \mathrm{C}$ with a three times change of osmic acid [14]. After fixing the slices were washed in $0.1 \mathrm{~mol} / \mathrm{L}$ phosphate buffer, $\mathrm{pH} 7.4$, dehydrated in increasing concentrations of ethanol, absolute acetone or propylene oxide with preliminary postfixation and contrast with a saturated solution of uranyl acetate and sealed in a mixture of epoxy and araldite. Half-thin sections of $1 \mu \mathrm{m}$ thickness were stained with a $1 \%$ solution of toluidine blue for light optical study and orientation of the selected area for the next electron microscopic evaluation. Ultra-thin sections were prepared with the help of ultra-thinner "LKB" and studied using an electron microscope "Philips $400 \mathrm{~T}$ " at an accelerating voltage of $80 \mathrm{kV}$.

\section{RESULTS AND DISCUSSION}

We have previously shown that rats develop ultrastructural changes in the skin 30 days after modeling portal hypertension. We will not repeat the identified features, but present the results of studies 60 and 90 days after the simulation of portal hypertension, which will allow us to characterize the dynamics of ultrastructural changes in the skin.

60 days after the start of the experiment, the electron microscopy revealed severe edema of the basal and spinous layer of the epidermis, breakdown of intercellular contacts. Loci of increased density were bordering with the transparent zones of different shapes and volume. The intercellular spaces at the same time looked expanded, had signs of desmosomes destruction.

Changes in the basement membrane of the epidermis were manifested in its thickening, homogenization, the formation of areas that did not have their transparent zone. Transparent area between basal epidermis and the basal membrane of the epidermis was deformed, areas of its expansion were detected, cavities were formed that displaced the basal membrane from basal epidermis. The cells of the basal and spinous layers were reduced in size.

Cytolemma of the basal epithelium was preserved, but on the lateral sites it was poorly visualized, in these areas there were loci of a destroyed cytolemma. The nuclei of basal epidermis were weakly shaped, had a condensed and unevenly distributed chromatin, wavy and tooth-like contours. Around the nuclei of basal epidermis there were transparent zones (perinuclear edema). The density of the cytoplasm of basal epidermis was associated with an increase in the number of tonofibrils, which looked massive, condensed, and rough. The morphology of mitochondria acquired differences that manifested in the destruction and disturbance of the spatial orientation of cristae, changes in matrix density (from very light to dark). Degenerative forms appeared in the form of dark electron-density granules of round or elongated shape. Other elements of the cytoplasm ultrastructure turned into polymorphic granules with undetermined shape. In the cytoplasm, in addition to intact organelles, inclusions appeared in the form of polymorphic granules of different densities, which corresponded to the locus of its destruction. The number of free ribosomes was reduced, they were chaotically scattered by the cytoplasm. Transparent intranuclear edging, bordering with fenestrated nuclear membrane, was indicative of the nucleus swelling.

In the spinous layer of the epidermis, electron-transparent cavities were detected, which bordered with epidermocytes that had signs of vacuolar degeneration and destructive changes in ultrastructure. Most cells contained cytoplasm of rough and dense tonofibrils, which bordered with a weakly-formed nucleus. Indurations that arose because of condensation of thick and rough tonofibrils, as well as many dense and loose polymorphic granules were a sign of a destroyed ultrastructure. Collagen fibrils of the papillary layer of the dermis were intact. Only in the reticular layer were observed loci of their homogenization and formation of fibrosis sites. A decrease in the lumen of capillaries due to edema of endothelial cells was noted (Fig. 1).

The lesions of the microvessels of the dermis were manifested by their narrowing, perivascular edema and leukocyte infiltration, thrombosis. Endothelial cells with hyperosmic or sharply enlightened cytoplasm were found. The latter had swelled mitochondria and lysosomes. Cytolemma had a locus of desquamation and was convoluted on the luminous surface. The nuclei looked enlightened, they had fragmentation zones, the chromatin parts were located eccentrically, which indicated on the signs of apoptosis. The cytoplasm had a significant number of chaotically located ribosomes and vacuoles and enlargement of the perinuclear zone. The Golgi complex and the endoplasmic reticulum were poorly expressed. The latter had a reduced number of granules. Micropinocytic vesicles were located near the basal and luminous surfaces of the capillaries. The cytoplasm of fibroblasts contained a significant number of polymorphic vacuoles and inclusions, mitochondria with a transparent matrix that had signs of edema with areas of membrane destruction and locus of unstructured dense material (destroyed cristae) (Fig. 2).

Tissue basophils were located near the capillaries, contained a moderate number of vacuoles and granules of average electron density. Their cytolemma in many loci was not detected. 


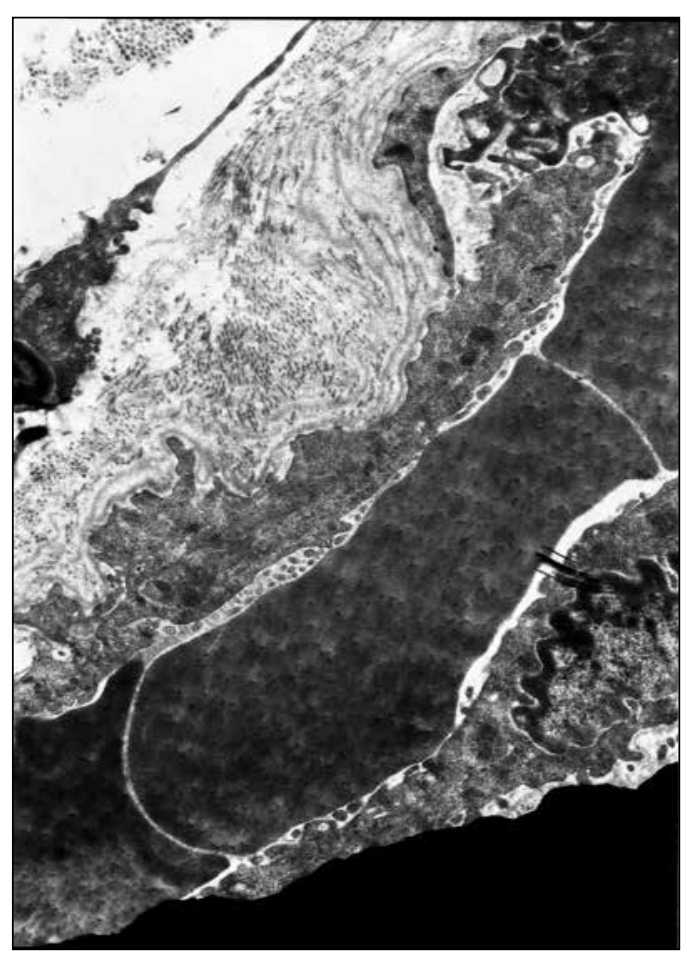

Fig. 1. Adecrease in the lumen of capillaries due to edema of endothelial cells.

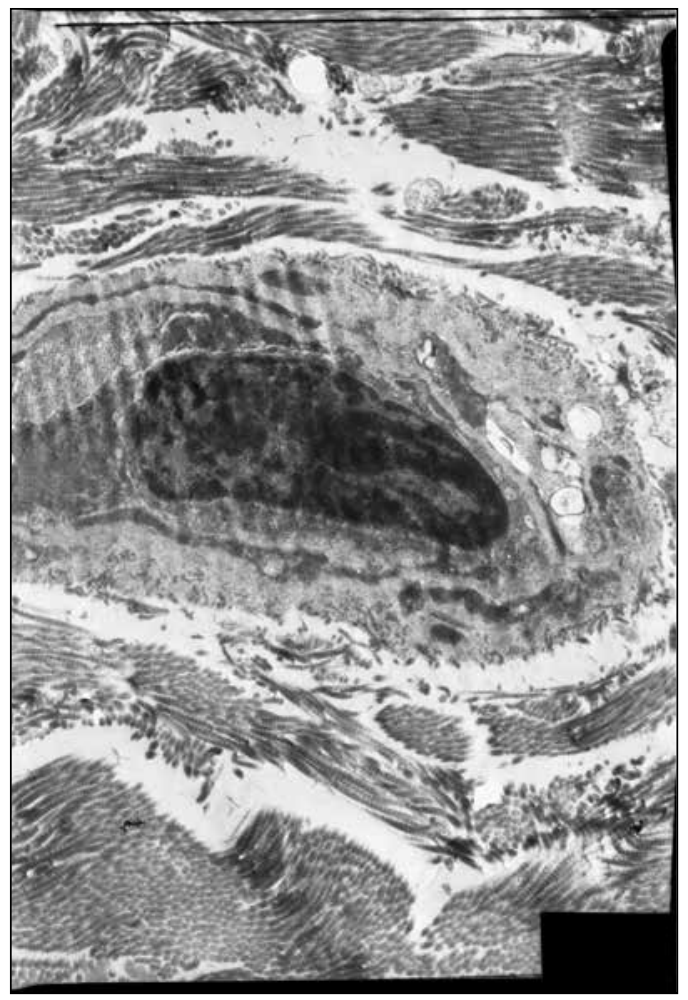

Fig. 3. Visualization of the fibroblast ultrastructure

90 days after the experiment beginning, the basal membrane of the epidermis was thickened and tightened, its transparent area was deformed and at some loci disappeared. Basal epidermocytes were enlightened given a deeply destroyed structure, had signs of perinuclear edema and condensation of chromatin. In the intercellular spaces

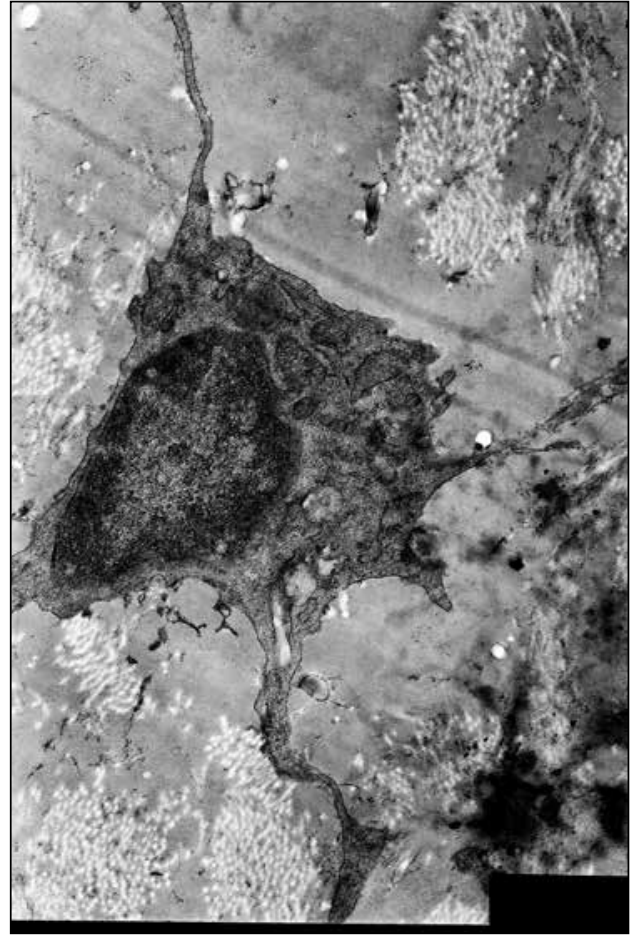

Fig. 2. Destroyed cristae

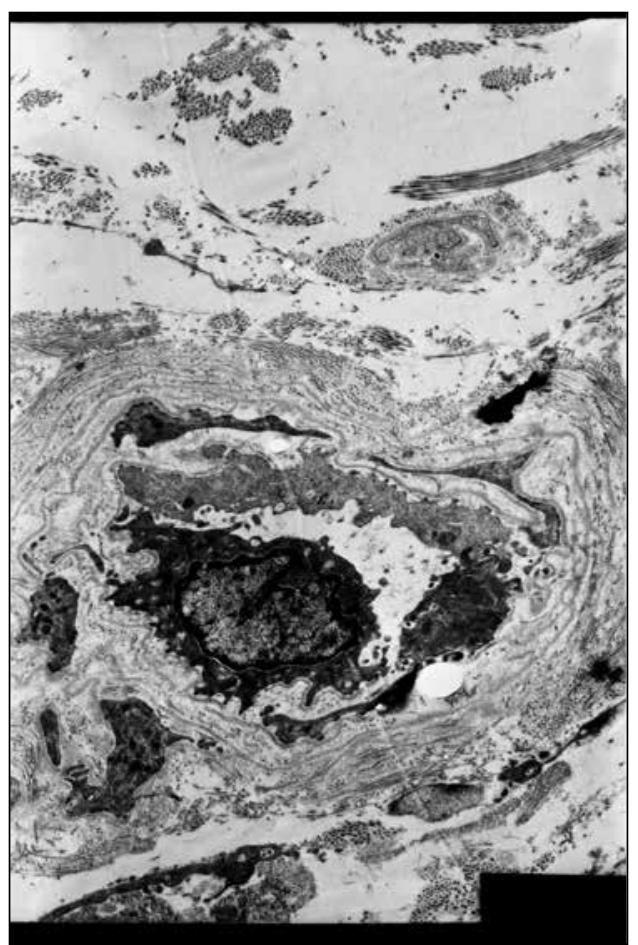

Fig. 4. Fibroblasts containing the nucleus which occupied more than half of the cell volume

there were cavities that corresponded to the intercellular edema locus. Tonofibrils of basal epidermis were tough and condensed, mitochondria - partially destroyed and polymorphic, looked like a tight oval cell, contained fragments of cristae, tonofilaments were not detected, ribosomes had a chaotic location along the cytoplasm, and formed groups 
of 4 to 8 units. Their cytolemma was badly contoured, however, the desmosomes were of a typical structure. In some epidermocytes the spinous layer of the cytoplasm was destroyed. Instead, a small and large grain electron negative material was observed, as well as thick bundles of tonofibrils. The nucleus density arose due to condensation of chromatin, the loci of karyolemma degradation and intranuclear inclusions were detected due to intranuclear cytoplasm invagination. Among the dense polymorphic material of the destroyed cytoplasm ultrastructure, there were many transparent areas, that was a sign of cytoplasmic edema. Intercellular spaces were significantly expanded, with a significant number of desmosomes. The cytoplasm of the granular layer epidermocytes contained a significant number of grouped dense granules, resembling ribosomes, a moderate amount of hard-fibrous dense material. Desmosomes were slightly contoured. The spinous layer lost communication with the underlying structures. In epidermocytes of the spinous and granular layers, processes of keratinization have been violated. Weak visualization of the fibroblast ultrastructure was associated with expansion of the endoplasmic reticulum tanks, illumination of the nucleus and its intranuclear edema, nuclear membrane lysis areas, swelling of mitochondria, which gained great enlightened form of petals with round or oval form (Fig. 3).

However, fibroblasts containing the nucleus were detected, which occupied more than half of the cell volume, had a well-developed lamellosa complex, high secretory activity. Ultrastructural changes of microvessels were characterized by narrowed lumen. Endothelial cells had significant edema and moderate vacuolization of the cytoplasm. Cell's nuclei were of normal volume, but chromatin was indurated (Fig. 4). In the cytoplasm there was a moderate amount of chaotically located polysomes and free ribosomes. The mitochondria of the endothelial cells had a slightly hyperosmic matrix and deformed cristae, there was a moderate number of young and gigantic forms. Granular endoplasmic reticulum was well developed, but the number of ribosomes on it was reduced. Erythrocytes diapedesis and sometimes microhemorrhages were noted, in the pericapillary space a significant number of leukocytes and red blood cells were detected. The collagen fibers of the dermis preserved their general structure, but were partially fragmented, swollen, had an enlarged diameter. Their dissociation into individual fragments of irregular shape occured. Some amount of immature collagen fibers merged into the depths of an amorphous substance. Elastic fibers looked intact. Tissue basophils looked smaller in volume, containing single granules and many large vacuoles. The proportion between dark and light cells was changed significantly: a prominent increase in the number of dark cells and a decrease in light ones was noted, the most pronounced changes were detected in the hypodermis. A destruction of nerve fibers in the form of their varicose, vacuolization, deep and granular decay of axial cylinders was noted, that was expressed most of all on free nerve endings.

So, changes in the endothelial cells of the dermis papillary layer microvessels, noted in portal hypertension, is a response to the direct action of elevated pressure in the portal vein and is a secondary response of microvessels to systemic vascular damage.

\section{CONCLUSION}

1. 60 days after modeling portal hypertension we established the development of severe edema of the basal and spinous layer of the epidermis, breakdown of intercellular contacts. Changes in the basement membrane of the epidermis were manifested in its thickening, homogenization, the formation of areas that did not have their transparent zone. In the spinous layer of the epidermis the sighns of vacuolar degeneration and destructive changes in ultrastructure were detected. The lesions of the microvessels of the dermis were manifested by their narrowing, perivascular edema and leukocyte infiltration, thrombosis.

2. 90 days after the simulation of portal hypertension, changes in ultrastructure and microcirculation increased and were most pronounced during all observation periods.

3. The obtained results should be taken into account in patients with portal hypertension and arterial hypertension when performing operations, as neglect of them can lead to complications.

\section{REFERENCES}

1. Romeo P, Lavanga V, Pagani D, Sansone V. Extracorporeal Shock Wave Therapy in Musculoskeletal Disorders: A Review. Med Princ Pract. 2014;23:7-13.

2. Berman B, Maderal A, Raphael B. Keloids and Hypertrophic Scars: Pathophysiology, Classification, and Treatment. Dermatol Surg. 2017 Jan;43:3-18.

3. Ojeh N, Bharatha A, Gaur U, et al. Keloids: Current and emerging therapies. Scars Burn Heal. 2020; 6:2059513120940499. doi: 10.1177/2059513120940499.

4. Maricevich MA, Adair MJ, Maricevich RL, et al. Facelift complications related to median and peak blood pressure evaluation. Aesthetic Plast Surg. 2014; 38(4):641-7.

5. Park DM. Total Facelift: Forehead Lift, Midface Lift, and Neck Lift. Arch Plast Surg. 2015;42(2):111-125.

6. Cason RW, Avashia YJ, Shammas RL, et al. Perioperative Approach to Reducing Hematoma during Rhytidectomy: What Does the Evidence Show? Plastic and Reconstructive Surgery. 2021;147(6):1297-1309.

7. Baker DC, Stefani WA, Chiu ES. Reducing the Incidence of Hematoma Requiring Surgical Evacuation following Male Rhytidectomy: A 30-Year Review of 985 Cases. Plastik and Reconstructive Surgery. 2005;116(7):1973-1985.

8. Moreno M, Bataller R. Cytokines and renin-angiotensin system signaling in hepatic fibrosis. Clin Liver Dis. 2008;12(4):825-852.

9. Niazi F, Hooshyar SH, Hedayatyanfard K, et al. Detection of Angiotensin II and AT1 Receptor Concentrations in Keloid and Hypertrophic Scar. J Clin Aesthet Dermatol. 2018;11(10):36-39.

10. lannello S, Milazzo P, Bordonaro F, et al. Low-dose enalapril in the treatment of surgical cutaneous hypertrophic scar and keloid-two case reports and literature review. MedGenMed. 2006;8(4):60.

11. Mirabito Colafella KM, Bovee DM, Danser AHJ. The renin angiotensin aldosterone system and its therapeutic targets. Exp Eye Res. 2019;186: 107680. 
12. Schwabl P, Brusilovskaya K, Supper P, et al. The soluble guanylate cyclase stimulator riociguat reduces fibrogenesis and portal pressure in cirrhotic rats. Sci Rep. 2018 Jun 19;8(1):9372.

13. Königshofer P, Brusilovskaya K, Schwabl P, Reiberger T. Animal models of portal hypertension. Biochim Biophys Acta Mol Basis Dis. 2019 May1;1865(5):1019-1030.

14. Sarkisov DS, Perov YuL, editors. Microscopic technique: a guide. Moscow: Meditsina; 1996. 544 p.( Russian).

The work was performed in the framework of research work "Development of new soft dressings and methods of their application in the treatment of wounds", which was funded by the Ministry of Education and Science of Ukraine, state registration № 0118 U002056.

\section{ORCID and contributionship:}

Nazar R. Hrytsevych: 0000-0002-9627-2099 B, C, D, F

Volodymyr V. Vereschaka: 0000-0003-0978-8028 A,E

\section{Conflict of interest:}

The Authors declare no conflict of interest.

\section{CORRESPONDING AUTHOR}

\section{Nazar R. Hrytsevych}

Striletska street, 10, Lviv, 79014, Ukraine

tel: +380677131940

e-mail:hrytsevych@gmail.com

Received: 30.06 .2021

Accepted: 25.08 .2021

A - Work concept and design, B - Data collection and analysis, C - Responsibility for statistical analysis, D -Writing the article, $\mathbf{E}$-Critical review, $\mathbf{F}$ - Final approval of the article 